\title{
Magnetic molecularly imprinted stirring bar for isolation of patulin using grafting technique
}

\author{
M. Díaz-Bao, *P. Regal, R. Barreiro, J.M. Miranda, A. Cepeda \\ Department of Analytical Chemistry, Nutrition and Bromatology, Faculty of Veterinary Science \\ (Lugo), University of Santiago de Compostela \\ * Corresponding e-mail: patricia.regal@usc.es
}

\begin{abstract}
Mycotoxins are low-molecular-weight natural products with great structural diversity produced as secondary metabolites by fungi. One of the principal toxic fungal metabolites is patulin, produced by over 30 genera of mold including species as Penicillium expansum or Penicillium griseofulvum, and normally related to vegetable-based products and fruit, mainly apple. These molds grow easily in damaged fruit or in derived products as juices if storage conditions are deficient. Some of the most serious effects of patulin ingestion are agitation, convulsions, edema, ulceration intestinal, inflammation and vomiting. Thus, European Regulation 1881/2006 established a maximum content of $10 \mathrm{ppb}$ in infant fruit juices, $50 \mathrm{ppb}$ for fruit juices in adults and $25 \mathrm{ppb}$ in fruit-derived products. Nowadays, the official analytical method adopted by AOAC International is HPLC with UV detection, using clean-up with ethyl acetate and sodium carbonate. However, the diverse drawbacks of this method (poor stability of patulin under alkaline extraction, poor resolution between patulin and co-extracted hydroxymethylfurfural) have originated interest in alternative options, such as LC methods coupled to mass spectrometry. In the last years, purification with molecularly imprinted polymers started to be used and they are becoming promising materials in analytical chemistry. In the present work, a selective magnetic molecularly imprinted stir-bar has been developed for the rapid isolation of patulin. A structural analogue, 2-oxindole, was used as dummy template. The polymer was grafted to the silanized glass surface of the stir-bar.
\end{abstract}

Keywords: patulin; MMIP; stir-bar; grafting; HPLC-MS/MS; apple 


\section{Introduction}

Mycotoxins are low-molecular-weight natural products with great structural diversity produced as secondary metabolites by fungi. Patulin (PAT) is an important toxic metabolite or mycotoxin produced by over 30 genera of mold such as Penicillium, Aspergillus, and Byssochlamys. Particularly, Penicillium expansum is known as the main source of PAT and it is commonly associated with apple rot (Desmarchelier et al., 2011). Although, PAT is mainly detected in apples and derived products, it is also found in barley, wheat, corn, silage, pears, grapes and peaches (Smet et al., 2011). These molds grow easily in damaged fruit or in derived products as juices, if storage conditions are deficient. Some of the most serious health effects of PAT ingestion in humans are agitation, convulsions, edema, intestinal ulceration, inflammation and vomiting (Silva et al., 2007). The toxicity of these molecules has led to the setup of strict regulations in many countries for their control in food and feed, and the consequent establishment of official legislation. The establishment of maximum limits in some food products resulted in an increasing demand of sensitive, selective and effective analytical methods (Pires et al., 2012). Thus, the European Commission Regulation (EC) 1881/2006 established a maximum content for PAT of $10 \mathrm{ppb}$ in infant fruit juices, $50 \mathrm{ppb}$ for fruit juices in adults and $25 \mathrm{ppb}$ in fruit-derived products (EC, 2006).

For the determination of PAT, thin layer chromatography was firstly used. Nowadays, the official analytical method adopted by the Association of Official Analytical Chemists (AOAC) for the analysis of PAT in food is HPLC with UV detection, using an extraction with ethyl acetate and clean-up with sodium carbonate (Brause et al., 1996). The main drawback of UV detection is the poor resolution between PAT and other co-extracted compounds such as hydroxymethylfurfural. As an additional problem, PAT is vulnerable to the alkaline conditions of the previously mentioned extraction method. Purification by solid-phase extraction (SPE) has been frequently applied as an alternative procedure. More recently, LC methods coupled to mass spectrometers are preferred in routine because of their great selectivity and sensitivity (Moake et al., 2005). In the last years, molecularly imprinted polymers (MIPs) started to be used, and are becoming promising materials for extracting different analytes present in food (Díaz-Bao et al., 2015; Regal et al., 2012). Currently, SPE using MIPs (MISPE) is extensively used for the extraction of various biologically active compounds. However, mycotoxins are usually too toxic or too expensive to be used as template molecules in MIP preparation. Template "bleeding" may be an additional problem of these polymers, especially when dealing with very low detection levels. In the present work, a rapid and selective method based on an in-house designed magnetic molecularly imprinted stir-bar (MMISB) 
has been developed for the isolation of PAT. For MIP synthesis, a structural analogue of PAT, 2oxindole, was used as dummy template (Figure 1) (Khorrami \& Taherkhani, 2011; Smet et al., 2011). The molecularly imprinted polymer was grafted on the silanized surface of a glass-covered stir-bar using an adaptation of typical protocols used in grafting techniques. The applicability of this novel stirring bar for the extraction of PAT has been tested in spiked of apples using HPLC-MS/MS for detection.

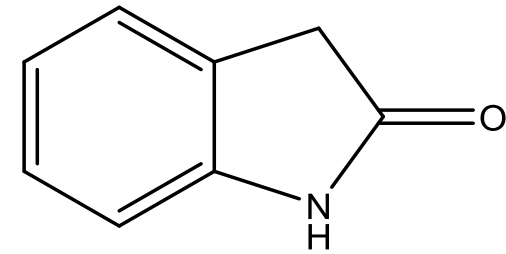

2-Oxindole

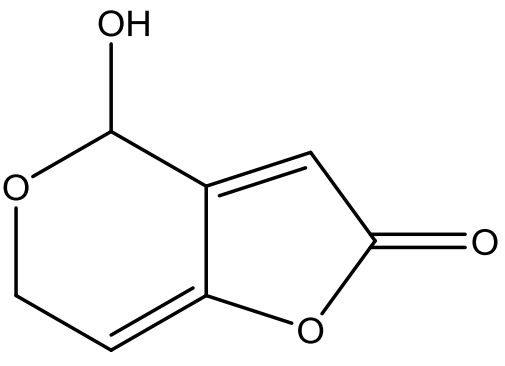

Patulin

Figure 1: Chemical structures of dummy template and patulin.

\section{Experimental}

\section{Materials}

The standard for PAT was purchased from Sigma-Aldrich Chemical Company (Madrid, Spain). PAT was suspended in methanol, resulting in the desired concentration for the analysis. The chemicals used for the polymers synthesis were 2-oxindole as dummy template, methacrylic acid (MAA), divinylbenzene 80\% (DVB-80), ethylene glycol dimethacrylate (EGDMA), and the initiator 2,2'- azobis-(2-methyl-butyronitril) (AIMN) from Sigma-Aldrich. MAA and EGDMA were freed from stabilizers by distillation under reduced pressure and AIMN was recrystallized from methanol prior to use. DVB-80 was freed from stabilizers by passing through a small column packed with neutral alumina (Aldrich). The 3-(methacryloxy) propyltrimethoxysilane was purchased from Sigma-Aldrich Chemical Company. HPLC grade solvents were supplied by Merck (Madrid, Spain).

\section{Apparatus}

These polymerization mixtures were simultaneously introduced into a temperature controllable incubator (Stuart Scientic, Redhill, Surrey, UK) at $60^{\circ} \mathrm{C}$ for 12 hours. The analyte recoveries were 
calculated using HPLC-MS/MS. Separation was performed in an 1100 series HPLC system from Agilent Technologies (Minnesota, USA). A Luna $3 \mu \mathrm{m} \mathrm{C18}(150$ × $2 \mathrm{mmm}$ ) column from Phenomenex (Torrance, CA, USA) was used. The mobile phase was water and methanol with $0.1 \%$ formic acid, mixed in isocratic mode at $70 \%$ and $30 \%$, respectively; the analytical run lasted for 10 min at $250 \mu \mathrm{L} / \mathrm{min}$. A Q-Trap 2000 mass spectrometer with ESI Source from AB Sciex (Toronto, Canada) was used, working in negative mode for PAT and in positive mode for 2-oxindole. For quantification of PAT, the most intense MRM transition was monitored along with a second transition for identity confirmation (Table 1).

Table 1. MRM transitions monitored for PAT detection in ESI negative mode, using HPLC-MS/MS.

\begin{tabular}{ll}
\hline MS/MS transition $(\mathbf{m} / \mathbf{z})$ & Used for \\
\hline $153>109$ & Quantitation \\
$153>81$ & Confirmation \\
\hline
\end{tabular}

\section{Design of molecularly imprinted stir-bars (MMISB)}

To achieve a stir-bar grafted with molecularly imprinted polymer on its surface, a chemical coating protocol adapted from the work of Turiel and Martin-Esteban was used (Turiel \& Martín-Esteban, 2012). First, a commercial glass-covered magnetic stir-bar remained in a combination of methanol and hydrochloric acid $(1: 1, \mathrm{v} / \mathrm{v})$, stirring for $30 \mathrm{~min}$ for clean-up of the glass surface. Next, the surface was silanized with $2 \% 3$-(methacryloxy) propyltrimethoxysilane in toluene for 1 hour (Cras et al., 1999). Finally, the stir-bar was rinsed methanol and let dry under $\mathrm{N}_{2}$ stream.

Once the silanized glass surface was ready, the chemical coating process took place as follows. In a glass tube of $4 \mathrm{~mL}$, the bar was placed and covered with the pre-polymerization mixture (Table 2). The stirring bar immersed in the monomeric recipe was allowed to polymerize at $60{ }^{\circ} \mathrm{C}$ for 12 hours, or until the appearance of the polymer was completely white. After (bulk) polymerization, the glass tube was broken to release the polymer-coated stir bar (Figure 2). Then, the bars were placed in a beaker and covered with water and methanol $(50 / 50, \mathrm{v} / \mathrm{v})$ to test the adhesion to the surface and the resistance of the polymer, using a magnetic stirrer. Next, the template molecule was removed by Soxhlet extraction for 12 hours with methanol/acetic acid (1/1) solution. To optimize the conditions of use of this MMISB for the extraction of PAT, different loading, washing and 
elution solution were tested. A magnetic non-imprinted stir bar (MNISB) was prepared in parallel, without the addition of template.

Table 2. Pre-polymerization mixture used to elaborate molecularly imprinted stir-bars and non-imprinted bars (no template addition) for PAT extraction.

\begin{tabular}{lll}
\hline \multicolumn{3}{c}{ PRE-POL } \\
\hline Function & Component & Ratio \\
\hline Dummy template & 2-oxindole & 1 \\
Functional monomer & MAA & 4 \\
Cross-linker & EGDMA & 20 \\
\hline Function & Component & Proportion \\
\hline Porogen & Toluene/Methanol (90:10) & $40 \% \mathrm{wt}$. \\
Initiator & AIMN & $2 \% \mathrm{wt}$. \\
\hline
\end{tabular}

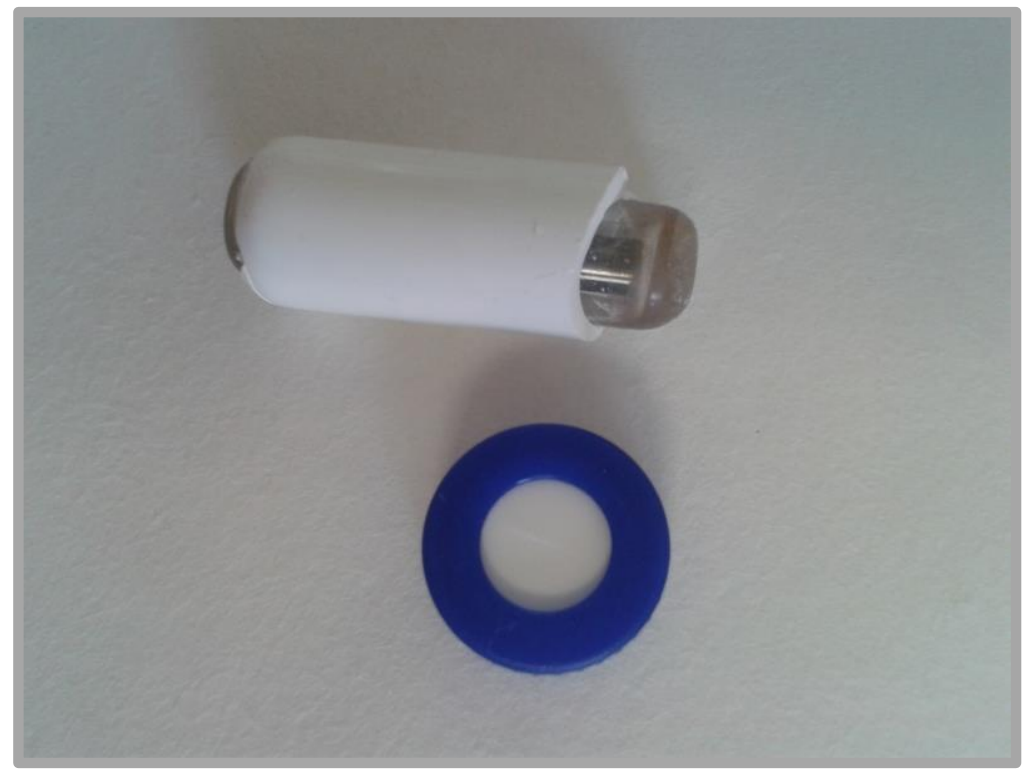

Figure 2: Magnetic molecularly imprinted stir bar (MMISB) developed for the extraction of patulin; the screw cap was placed below the stir-bar to illustrate its size.

\section{Application to real samples}

The optimized MMISB extraction protocol started adding water to cover $3 \mathrm{~g}$ of diced apple in a 50 $\mathrm{mL}$ tube. Apple samples were spiked with water containing PAT at $50 \mathrm{ppb}$ (MRL to for fruit juices in adults) and introduced into an ultrasound bath for $30 \mathrm{~min}$. Samples were shaken for $10 \mathrm{~min}$, 
centrifuged at 5,000 $\mathrm{g}$ for $10 \mathrm{~min}$ and the supernatant was transferred to a baker with the stir bar. A conventional SPE protocol was applied, consisting of loading, washing and elution steps with different solvents. The analyte was loaded within the pores (active sites) of the MMISB under stirring for $30 \mathrm{~min}$. After this sorptive extraction, water was removed with the aid of external magnet and stir-bar was washed with water for $5 \mathrm{~min}$. Then, to elute the retained patulin in MMISB, $5 \mathrm{~mL}$ methanol/acetic acid (75/25) were added and kept stirring for $30 \mathrm{~min}$. Finally, the eluate was evaporated under nitrogen stream at $30^{\circ} \mathrm{C}$ and re-dissolved in $100 \mu \mathrm{L}$ of mobile phase. Twenty microliters were immediately injected into the chromatographic system for analysis. Recoveries were calculated using HPLC-MS/MS.

\section{Results and discussion}

A structural analogue of patulin, 2-oxindole, was used as a dummy template to synthesize a MIP capable of selectively binding the molecule of PAT (Khorrami \& Taherkhani, 2011). The obtained cavities in the prepared MIP are supposed to have a complementary shape and electronic affinity to the template. This dummy-template approach was preferred to minimize the manipulation of patulin, because this type of analytes can be hazardous to the personnel working in the laboratory. The selected polymerization technique was bulk polymerization, which it does not require sophisticated instrumentation and because the reaction conditions can be easily controlled. Furthermore, it is the most widely used method for the preparation of polymers (Regal et al., 2012).

In the design of any pre-polymerization mixture, the selection of cross-linker and porogen solvent are two key factors. These two components would determine the aspect, strength and even the color and porosity of the final polymer. DVB-80 and EGDMA, two of the most common cross-linkers used for MIP synthesis, were tested in combination with various combinations of toluene with methanol and/or acetonitrile as porogenic solvent. The different combinations of monomer-solvent were introduced in an injection vial and let polymerize under bulk conditions for 24 hours at $60{ }^{\circ} \mathrm{C}$. After polymerization, the glass vials were broken and the resulting material was tested visually and by touching. With the use of DVB-80 as cross-linker, it was impossible to obtain a strong and tough polymer. The resulting polymer would be a plastic, yellowish, cracked and fragile material, not suitable for resisting the required stirring conditions. Thus, adequate divinylbenzene polymers could not be achieved using the different porogens solvents (acetonitrile or methanol), even changing the toluene percentage (from 0 to $90 \%$ toluene). As for EGDMA, the polymers were in every case stronger and wither than those obtained with DVB, showing more resistance to compressive strength and even more when using methanol (combined with toluene) instead of acetonitrile. The 
mixture of methanol and toluene (90:10) with EGDMA proved to be useful, resulting in a hard and white polymer. Consequently, this combination was used for MMISB preparation.

Then, the efficiency of the obtained polymer was evaluated with a very simple extraction protocol, for the isolation of PAT from apple samples prior to analysis. The protocol consisted simply on pouring-in, stirring and pouring-out solvents from a beaker. To carry out the extraction process, it was only necessary to have a magnetic stirrer and an external magnet. Satisfactory recoveries of PAT were obtained using this molecularly imprinted glass-covered stir bar, with $60-70 \%$ of recovery in a minimum loading time of 45 min under stirring. Besides, the deterioration of the polymeric coat was imperceptible after several hours of use, indicating that the adhesion of the polymer was strong and stable. The glass layer as cohesion agent between the bar and the polymeric coat can be assumed. NIP stir bars showed a mean recovery difference of $20 \%$ less.
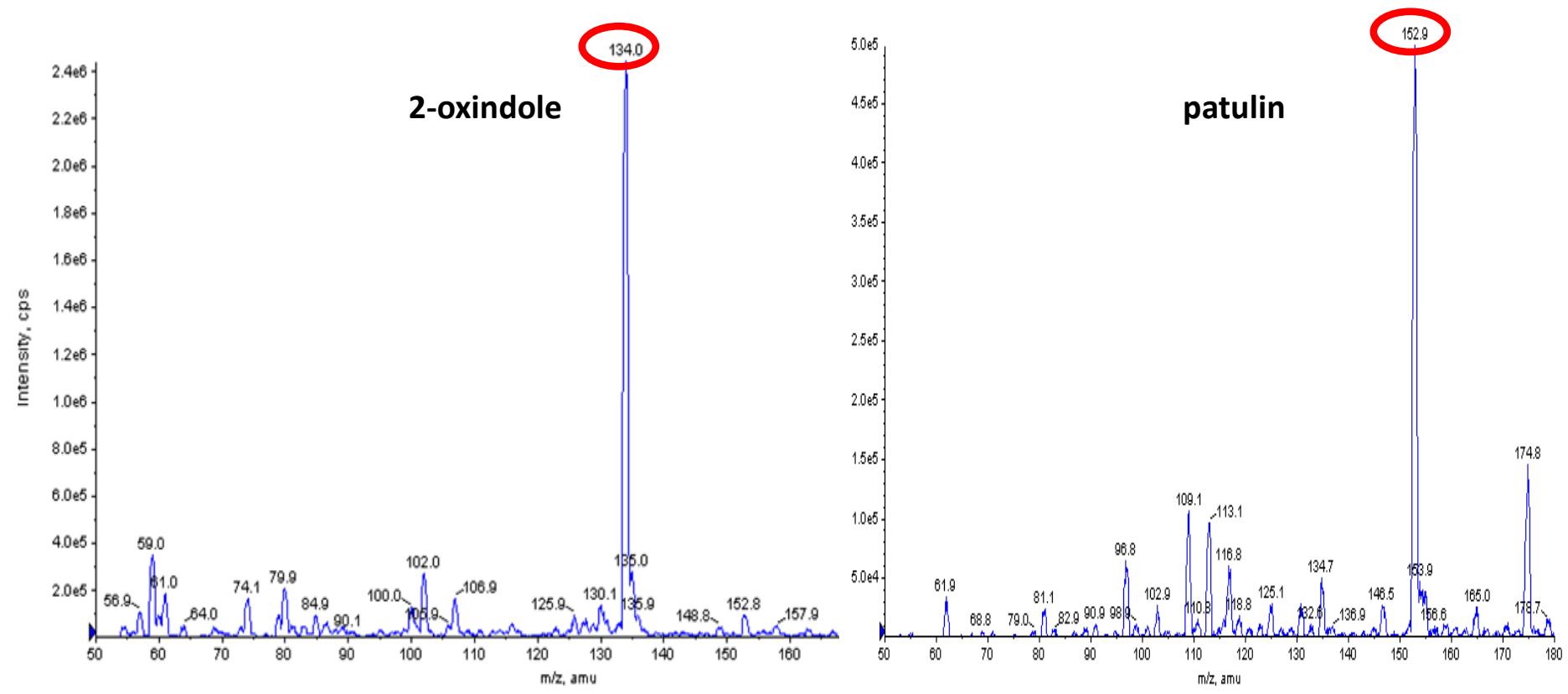

Figure 3. MS spectrum obtained from a standard solution of PAT infused in the MS using ESI negative mode, and 2-oxindole using ESI positive mode, showing the structural similitudes.

\section{Conclusions}

The proposed magnetic extraction has demonstrated to be useful for the isolation of PAT in apple at $50 \mathrm{ppb}$. The stir-bars are easy to use and the extraction protocol was reduced to simply add and remove solvents from a beaker, with the aid of a magnetic stirrer and an external magnet. MIPs offer a broad range of cheap, versatile and selective pretreatment solutions for food analysis. The polymer coated on the surface of magnetic stir-bars was prepared in a non-covalent approach, using 
a dummy template to avoid "template bleeding" and to limit the exposure of laboratory staff to mycotoxins. In this case we selected 2-oxindole, structurally related to PAT molecule (Figure 3), as template for the design of a MIP selective towards PAT.

\section{Acknowledgements}

This research was supported by the Project for Emerging Researchers EM 2012/153 from Consellería de Cultura, Educación e Ordenación Universitaria (Xunta de Galicia).

\section{References}

Brause, A.R. et al. (1996) Determination of patulin in apple juice by liquid chromatography: collaborative study. Journal of AOAC International, 79 (2), 451-455.

Cras, J. J., Rowe-Taitt, C. A., Nivens, D. A., \& Ligler, F. S. (1999). Comparison of chemical cleaning methods of glass in preparation for silanization. Biosensors and Bioelectronics, 14(8-9), 683-688.

Desmarchelier, A., Mujahid, C., Racault, L., Perring, L., \& Lancova, K. (2011). Analysis of patulin in pear- and apple-based foodstuffs by liquid chromatography electrospray ionization tandem mass spectrometry. Journal of Agricultural and Food Chemistry, 59(14), 76597665 .

Díaz-Bao, M., Barreiro, R., Miranda, J. M., Cepeda, A., \& Regal, P. (2015). Recent advances and uses of monolithic columns for the analysis of residues and contaminants in food. Chromatography, 2, 79-95.

European Commission Regulation (EC) 1881/2006 setting maximum levels for certain contaminants in foodstuffs. Official Journal of the European Union L 364, 5-24.

Khorrami, A., \& Taherkhani, M. (2011). Synthesis and evaluation of a molecularly imprinted polymer for pre-concentration of patulin from apple juice. Chromatographia, 73(1), 151156.

Moake, M. M., Padilla-Zakour, O. I., \& Worobo, R. W. (2005). Comprehensive review of patulin control methods in foods. Comprehensive Reviews in Food Science and Food Safety, 4(1), $8-21$. 
Pires, S., Lopes, J., Nunes, I. and Gaspar, E. (2012). Patulin Analysis: Sample Preparation Methodologies and Chromatographic Approaches, Ecotoxicology, Dr. Ghousia Begum (Ed.), InTech.

Regal, P., Diaz Bao, M., Barreiro, R., Cepeda, A., Fente, C. (2012). Application of molecularly imprinted polymers in food analysis: Clean-up and chromatographic improvements. Central European Journal of Chemistry, 10(3), 766-784.

Silva, S. J. N., Schuch, P. Z., Bernardi, C. R., Vainstein, M. H., Jablonski, A., \& Bender, R. J.. (2007). Patulin in food: state-of-the-art and analytical trends. Revista Brasileira de Fruticultura, 29(2), 406-413.

Smet, D., Dubruel, P., Peteghem, V., \& Saeger, S. (2011). Development of a molecularly imprinted polymer for patulin in apple juice. World Mycotoxin J, 4, 375-383.

Turiel, E., \& Martín-Esteban, A. (2012). Molecularly imprinted stir bars for selective extraction of thiabendazole in citrus samples. Journal of Separation Science, 35(21), 2962-2969. 